\title{
MAP ESTIMATION OF THE INPUT OF AN OVERSAMPLED FILTER BANK FROM NOISY SUBBANDS BY BELIEF PROPAGATION
}

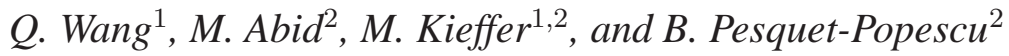 \\ ${ }^{1}$ L2S - CNRS - SUPELEC - Univ Paris-Sud, 91192 Gif-sur-Yvette, France \\ 2 Telecom ParisTech, Signal and Image Processing Department, \\ 46 rue Barrault, 75634 Paris cedex 13, France
}

\begin{abstract}
Oversampled filter banks perform simultaneously subband decomposition and redundancy introduction. This redundancy has been shown to be useful to combat channel impairments, when the subbands are transmitted over a wireless channel, as well as quantization noise. This paper describes an implementation of the maximum a posteriori estimator of the input signal from the noisy quantized subbands obtained at the output of some transmission channel. The relations between the input samples and the noisy subband samples are described using a factor graph. Belief propagation is then applied to get the posterior marginals of the input samples. The experimental results show that when the channel is clear, a least-squares estimate is satisfying. But, the proposed approach performs significantly better than a least-squares reconstruction when the channel is noisy: a gain in terms of channel SNR of more than $2 \mathrm{~dB}$ is observed.
\end{abstract}

\section{INTRODUCTION}

Recently a growing interest has been dedicated to communication systems performing jointly source and channel coding [2]. Such schemes cope better with unknown and changing channel characteristics than the classical tandem schemes. In this context, multirate systems and more particularly Oversampled Filter Banks (OFB) $[3,4]$ are attractive solutions since they provide an overcomplete representation of the input signal by introducing some structured redundancy among the output subbands. OFB may then be seen as errorcorrecting codes in the real field as evidenced in [6-8]. OFB may correct transmission errors left by channel decoders and mitigate a part of the quantization noise [9]. Specific decoding techniques have been developed for OFB. Hypotheses testing and maximum likelihood estimation are considered in [6]. Kalman filtering is considered in [8]. A consistent reconstruction technique accounting for the bounded nature of the quantization noise is introduced in [10].

This work considers the maximum a posteriori (MAP) estimation of the input of an OFB, when its output subbands are quantized and transmitted over a noisy channel. The computation of the exact MAP estimator is intractable in general, even for moderate-size input signals. When the OFB consists of finite impulse response filters, a factor graph may describe the relations between the input samples and the noisy subband samples. Belief propagation (BP) may then be used to compute the posterior probability distribution (PPD) of each entry of the input vector knowing the noisy subbands. This approach is inspired from $[11,12]$ where the problem of estimating some input vector $\mathbf{x} \in \mathbb{R}^{n}$ from noisy observations $\mathbf{y} \in \mathbb{R}^{m}$ of linear measurements $\mathbf{z}=\Phi \mathbf{x}$ of $\mathbf{x}$ has been addressed with BP. This problem is known as a linear mixing estimation problem. Via BP, the linear relations between the variables are exploited to update their
PPD. This is done by passing messages on the variable states along a graph [13-15]. This message passing algorithm (MPA) operating in real field is similar to MPA for LDPC codes which work in finite fields [16]. The exact implementation of BP for dense mixing matrices is computationally very complex as it involves high-dimensional integrations for the PPD calculation. Implementations of BP based on Gaussian approximations have proven to be efficient and accurate as for example the Generalized Approximate Message Passing (GAMP) algorithm [12].

When the length of the impulse response of the filters involved in the OFB is not too large, the $\Phi$ matrix associated to the OFB may be quite sparse. Approximate implementation of the BP algorithm using discretized probability density functions becomes then tractable and has been considered here.

The rest of the paper is organized as follows. The considered communication scheme is presented in Section 2. The link between input estimation of OFBs from noisy subbands and linear mixing estimation problems is detailed in Section 3. The MAP estimation using BP is then described in Section 4. Finally, preliminary experimental results are described in Section 5 before drawing some conclusions in Section 6.

\section{TRANSMISSION SCHEME}

The communication scheme considered here is depicted in Figure 1. The random input vector $\mathbf{x} \in \mathbb{R}^{n}$ has i.i.d. components with prior

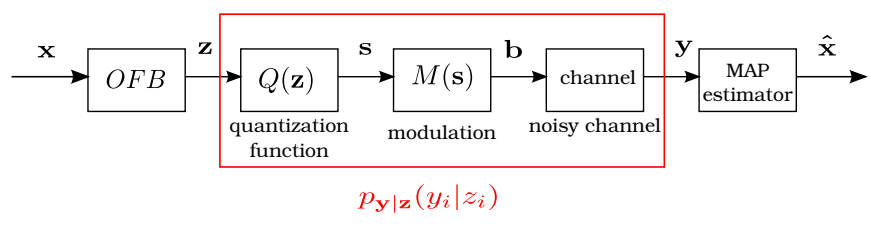

Fig. 1. Transmission scheme based on an OFB

probability density function (pdf) $p_{X}\left(x_{j}\right), j \in\{0, \ldots, n-1\}$. This vector passes first through an OFB introducing a redundancy $\rho=$ $m / n$. The resulting vector $\mathbf{z} \in \mathbb{R}^{m}$ is then quantized to get a vector of quantization indexes $\mathbf{s}$. The quantization function is denoted by $Q(\mathbf{z})$ and the modulation function by $M(\mathbf{s})$. The modulated sequence corresponding to $\mathbf{s}$ and denoted by $\mathbf{b}$ is transmitted over $\mathbf{a}$ memoryless channel. Finally the observation y of real (or complex) values is obtained at the output of this channel.

In the particular case of a scalar quantization with the same rate $R$ for each subband sample and a BPSK modulation, each quantized index $s_{i}, i \in\{0, \ldots, m-1\}$ of $\mathbf{s}$ is represented by a binary sequence 
$\mathbf{b}_{i}$ of $R$ elements and the observation $\mathbf{y} \in \mathbb{R}^{m \times R}$ is formed by $m$ vectors $\mathbf{y}_{i} \in \mathbb{R}^{R}$ representing the components $z_{i}$ of $\mathbf{z}$. The problem is then to evaluate the MAP estimate $\hat{\mathbf{x}}$ of $\mathbf{x}$ :

$$
\hat{\mathbf{x}}=\arg \max _{\mathbf{x} \in \mathbb{R}^{n}} p(\mathbf{x} \mid \mathbf{y})
$$

The exact estimation of $\hat{\mathbf{x}}$ is intractable in practice when considering high-dimensional input vectors. We show in the next section that this problem can be seen as a particular linear mixing problem for which a suboptimal solution can be evaluated using the BP algorithm.

\section{LINEAR MIXING PROBLEM}

\subsection{General Scheme}

A general linear mixing problem is presented in Figure 2. The vector $\mathbf{x}$ goes through an $m \times n$ matrix $\Phi$ :

$$
\mathbf{z}=\Phi \mathbf{x}
$$

The output vector $\mathbf{z}$ is then transmitted over a separable measurement channel characterized by its conditional probability $p_{\mathbf{Y} \mid Z}\left(\mathbf{y}_{i} \mid z_{i}\right)$ and delivering the measurements $\mathbf{y}$. Here the quantization and modulation operations, assumed to be separable are incorporated into the measurement channel. The difficulty in the estimation of $\mathbf{x}$ know-

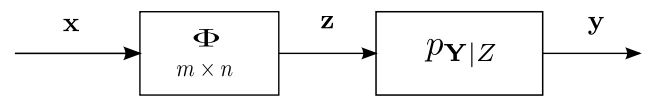

Fig. 2. General linear mixing estimation problem

ing $\mathbf{y}$ is that $\Phi$ mixes $\mathbf{x}$ to get $\mathbf{z}$. The evaluation of the posteriori pdf of each element $x_{j}, j \in\{0, \ldots, n-1\}$ or $z_{i}, i \in\{0, \ldots, m-1\}$ involves a high-dimensional integral that is difficult to evaluate. Such an estimation problem may be solved using BP, provided that a graph representing the dependencies between the variables is available. BP updates then the PPD of these variables via a message passing procedure along the edges of this graph $[13,16]$.

\subsection{OFB-based scheme}

An OFB is a filter bank whose number of output subbands is greater than the downsampling ratio. These subbands form then a redundant representation of the input signal. A typical $M-$ band OFB with a downsampling factor of $N \leq M$ such that $\rho=M / N$, is presented in Figure 3. This OFB is formed by $M$ FIR analysis fil-

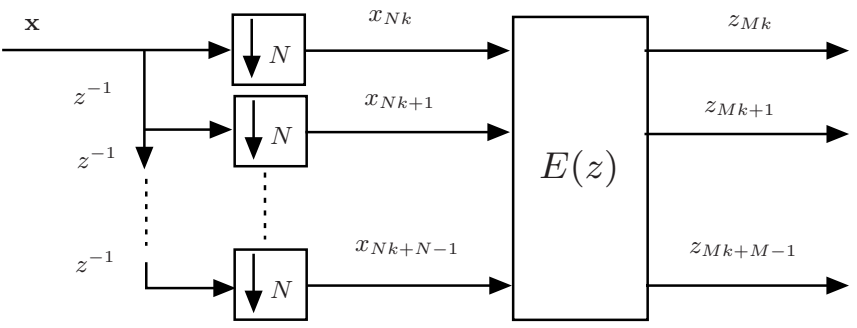

Fig. 3. Oversampled filter bank

ters $\left\{\mathbf{h}_{m}\right\}_{m=0}^{M-1}$ with maximal length $N \times(L+1)$. The polyphase representation of this OFB is the matrix:

$$
\mathbf{E}(z)=\sum_{l=0}^{L} \mathbf{E}_{l} z^{-l}
$$

where $\mathbf{E}_{l}, l=0, \ldots, L$ is a sequence of $M \times N$ matrices that can be constructed from $\left\{\mathbf{h}_{m}\right\}_{m=0}^{M-1}$ [17]. The following polyphase notations are used for the vectors $\mathbf{x}$ and $\mathbf{z}$ :

$$
\begin{aligned}
& \mathbf{x}=\left\{x_{0}, \ldots, x_{N-1}, \ldots, x_{k N}, \ldots, x_{k N+N-1}, \ldots, x_{n-1}\right\} \\
& \mathbf{z}=\left\{z_{0}, \ldots, z_{M-1}, \ldots, x_{k M}, \ldots, x_{k M+M-1}, \ldots, z_{m-1}\right\}
\end{aligned}
$$

where $k$ refers to the current instant. At each instant $k$ the input of the OFB is the vector $\mathbf{x}^{k}=\left(x_{N k}, \ldots, x_{N k+N-1}\right)^{T}$ and its output is the vector $\mathbf{z}^{k}=\left(z_{M k}, \ldots, z_{M k+M-1}\right)^{T}$ obtained as follows:

$$
\mathbf{z}^{k}=\sum_{l=0}^{L} \mathbf{E}_{l} \mathbf{x}^{k-l}=\mathbf{E}_{L: 0} \mathbf{x}^{k-L: k},
$$

where $\mathbf{x}^{k-L: k}=\left(\left(\mathbf{x}^{k-L}\right)^{T}, \ldots,\left(\mathbf{x}^{k}\right)^{T}\right)^{T}$ contains all input samples on which the OFB output at time $k$ depend and $\mathbf{E}_{L: 0}=\left(\mathbf{E}_{L}, \ldots, \mathbf{E}_{0}\right)$ is a $M \times(L+1) N$ matrix. One can then write the whole OFB operations as the linear mixing problem presented in (2), where

$$
\Phi=\left[\begin{array}{ccccccccc}
\mathbf{E}_{L} & . & . & . & . & \mathbf{E}_{0} & \mathbf{0} & \mathbf{0} & \mathbf{0} \\
\mathbf{0} & \mathbf{E}_{L} & . & . & . & . & \mathbf{E}_{0} & \mathbf{0} & \mathbf{0} \\
. & . & . & . & . & . & . & . & . \\
. & . & . & . & . & . & . & . & . \\
\mathbf{0} & . & \mathbf{0} & \mathbf{E}_{L} & . & . & . & . & \mathbf{E}_{0}
\end{array}\right]
$$

The MAP estimation problem formulated in (1) can then be solved using the BP algorithm.

\section{MAXIMUM A POSTERIORI ESTIMATION WITH BELIEF PROPAGATION}

Belief propagation is an iterative message passing algorithm [16] that associates to a transform matrix $\Phi$ a factor or Tanner graph $\mathcal{G}_{\Phi}$. An example of such a graph is presented in Figure 4. The graph $\mathcal{G}_{\Phi}$

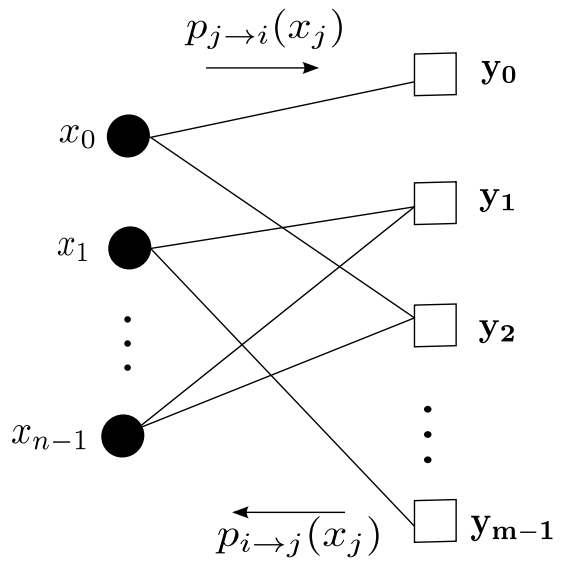

Fig. 4. Factor graph for the linear mixing estimation problem

is a bipartite graph formed by two kind of nodes: the variable nodes $j=0, \ldots, n-1$ corresponding to the input variables $x_{j}$ and the factor nodes $i=0, \ldots, m-1$ corresponding to the output measurements $\mathbf{y}_{i}$. An edge between the node $j$ and the node $i$ means that the entry $\Phi_{i j}$ is non-zero and thus the variables $x_{j}$ and $\mathbf{y}_{i}$ are involved in a linear relation. The set of variable nodes that are connected to the factor node $i$ is denoted by $N_{\text {out }}(i)$. Similarly the set of factor nodes 
connected to the variable node $j$ is denoted by $N_{i n}(j)$. The different nodes talk to each other by sending messages (beliefs) on the states of each input variable $x_{j}$ and the corresponding probabilities.

The message $p_{i \rightarrow j}\left(x_{j}\right)$ is sent by the factor node $i$ to the variable node $j$. It is a vector of the same dimension as the number of states in which $x_{j}$ can be. Each component of $p_{i \rightarrow j}\left(x_{j}\right)$ evaluates how likely the measurement $\mathbf{y}_{i}$ is obtained at node $i$ when the input variable $x_{j}$ belongs to the corresponding state. In a similar way, the message $p_{j \rightarrow i}\left(x_{j}\right)$ sent by $j$ to $i$ expresses the beliefs of the variable node $j$ about the states in which $x_{j}$ could be and their corresponding probabilities. When $\mathcal{G}_{\Phi}$ does not contain any cycle and after enough iterations, this series of message-passing is likely to converge to a consensus that determines the true marginal $p\left(x_{j} \mid \mathbf{y}\right)$.

The steps of the BP algorithm in real field are inspired by the ones presented by Rangan in [11]. They are resumed as follows:

1. Initialization:

(a) Set the current iteration $k=1$.

(b) For each variable node $j$ and factor node $i$ forming an edge of $\mathcal{G}_{\Phi}$ set the messages to the a priori distribution of the random variable $X_{j}$ :

$$
p_{j \rightarrow i}^{x}\left(k, x_{j}\right)=p_{j}^{x}\left(k, x_{j}\right)=p_{X_{j}}\left(x_{j}\right)
$$

2. Linear Mixing:

(a) Assume that the random variables $X_{j}$ are independent and that $X_{j} \sim p_{j \rightarrow i}^{x}\left(k, x_{j}\right)$

(b) Compute the distributions $p_{i \rightarrow j}^{z}\left(k, z_{i \rightarrow j}\right)$ and $p_{i}^{z}\left(k, z_{i}\right)$ of the random variables:

$$
Z_{i \rightarrow j}=\sum_{r \in N_{\text {out }}(i) \backslash j} \Phi_{i r} X_{r}
$$

and

$$
Z_{i}=\sum_{r \in N_{\text {out }}(i)} \Phi_{i r} X_{r}
$$

respectively.

\section{Output update:}

For each variable node $j$ and factor node $i$ forming an edge of $\mathcal{G}_{\Phi}$ compute the likelihood probability function

$$
p_{i \rightarrow j}^{u}\left(k, u_{i}\right)=P\left(\mathbf{Y}_{i}=\mathbf{y}_{i} \mid Z_{i}=Z_{i \rightarrow j}+u_{i}\right)
$$

evaluated on each point $u_{i}$.

\section{Input update:}

(a) For each variable node $j$ and factor node $i$ forming an edge of $\mathcal{G}_{\Phi}$ update the message sent by $j$ to $i$

$$
p_{j \rightarrow i}^{x}\left(k+1, x_{j}\right)=\alpha p_{X}\left(x_{j}\right) \prod_{l \in N_{i n}(j) \backslash i} p_{l \rightarrow j}^{u}\left(k, \Phi_{l j} x_{j}\right)
$$

where $\alpha$ is a normalization constant obtained by imposing that $p_{j \rightarrow i}^{x}\left(k+1, x_{j}\right)$ should sum up to 1 .

(b) For each variable node $j$ update the distribution

$$
p_{j}^{x}\left(k+1, x_{j}\right)=\beta p_{X}\left(x_{j}\right) \prod_{l \in N_{i n}(j)} p_{l \rightarrow j}^{u}\left(t, \Phi_{l j} x_{j}\right)
$$

where $\beta$ is a normalization constant obtained by imposing that $p_{j}^{x}\left(k+1, x_{j}\right)$ should sum up to 1 .

\section{Incrementation:}

(a) $k=k+1$

(b) Return to step 2. until a sufficient number of iterations is performed.

In order to estimate the input signal $\mathrm{x}$ of an OFB from its noisy received subbands $\mathbf{y}$, the direct implementation of this $\mathrm{BP}$ algorithm to perform the MAP estimation is possible as the correspondant matrix $\Phi$ is relatively sparse.

\section{EXPERIMENTAL RESULTS}

In this section we present the preliminary results obtained when using the MAP estimation based on the BP. We have considered an input vector $\mathbf{x} \in \mathbb{R}^{8}$. The components of $\mathbf{x}$ are i.i.d. zero-mean Gaussian with variance $\sigma_{x}^{2}=1$. The OFB used is based on the Haar filters with $M=6, N=4$, and $L=1$. The corresponding transform matrix $\Phi$ is

$$
\Phi=\left[\begin{array}{ccc}
\mathbf{E}_{1} & \mathbf{E}_{0} & \mathbf{0}_{6 \times 4} \\
\mathbf{0}_{6 \times 4} & \mathbf{E}_{1} & \mathbf{E}_{0}
\end{array}\right]
$$

where

$$
\mathbf{E}_{1}=\frac{1}{\sqrt{2}}\left[\begin{array}{cccc}
0 & 0 & 0 & 0 \\
0 & 0 & 1 & 1 \\
0 & 0 & 0 & 0 \\
0 & 0 & 0 & 1 \\
0 & 1 & 1 & 0 \\
0 & -1 & 1 & 0
\end{array}\right] \text { and } \mathbf{E}_{0}=\frac{1}{\sqrt{2}}\left[\begin{array}{cccc}
1 & 1 & 0 & 0 \\
0 & 0 & 0 & 0 \\
-1 & 1 & 0 & 0 \\
1 & 0 & 0 & 0 \\
0 & 0 & 0 & 0 \\
0 & 0 & 0 & 0
\end{array}\right]
$$

The vector $\mathbf{z} \in \mathbb{R}^{12}$ obtained at the OFB output is quantized using a scalar quantization function $Q(z)$ with a rate $R=4$ bits with a quantization step

$$
\Delta=\left(2 \sigma_{x}\right) /\left(2^{R}-1\right)
$$

Quantized samples are then BPSK modulated and transmitted over an AWGN channel with a SNR between $0 d B$ and $13 d B$. The number of noise realizations has been set to 1000 .

The MAP estimation using BP is compared to the reference least-squares (LS) approach.

\subsection{Reference estimation approach}

The reference estimation approach that is considered is presented in Figure 5. A classical decoder $D($.$) takes hard decisions on the$

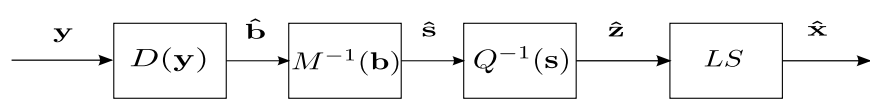

Fig. 5. Least Squares Estimation

received measurements $\mathbf{y}$. After demodulation and inverse quantization, the received vector $\hat{\mathbf{z}}$ is obtained. Finally the $\mathrm{LS}$ reconstruction is performed:

$$
\hat{\mathbf{x}}=\left(\Phi^{T} \Phi\right)^{-1} \Phi^{T} \mathbf{z}
$$




\subsection{Performances of the MAP estimation using BP}

The BP algorithm described in Section 4 is performed by considering probability mass functions approximating the continuous distributions. The range that has been considered for the input variables $x_{j}$ is from -10 to 10 . The number of points on which the probability distribution functions are evaluated has been set to 1024 . The considered resolution is then of $20 / 1024$. The total number of iterations of the BP algorithm is equal to 10. At each iteration, the messages $p_{j \rightarrow i}^{x}\left(k, x_{j}\right)$ and $p_{i \rightarrow j}^{u}\left(k, u_{i}\right)$ are vectors of 1024 entries where the probability distribution is evaluated.

The distribution computations in Step 2 are performed in two steps. First, the quantized distribution of the random variables $\Phi_{i r} X_{r}$ are computed using the fact that

$$
a X \sim \frac{1}{|a|} p_{X}(X / a)
$$

Then the convolution product is evaluated to determine the quantized distribution of the random variables $Z_{i \rightarrow j}$ and $Z_{i}$.

The experimental results that have been obtained are presented in Figure 6. One can see that the gain brought by the MAP estimation

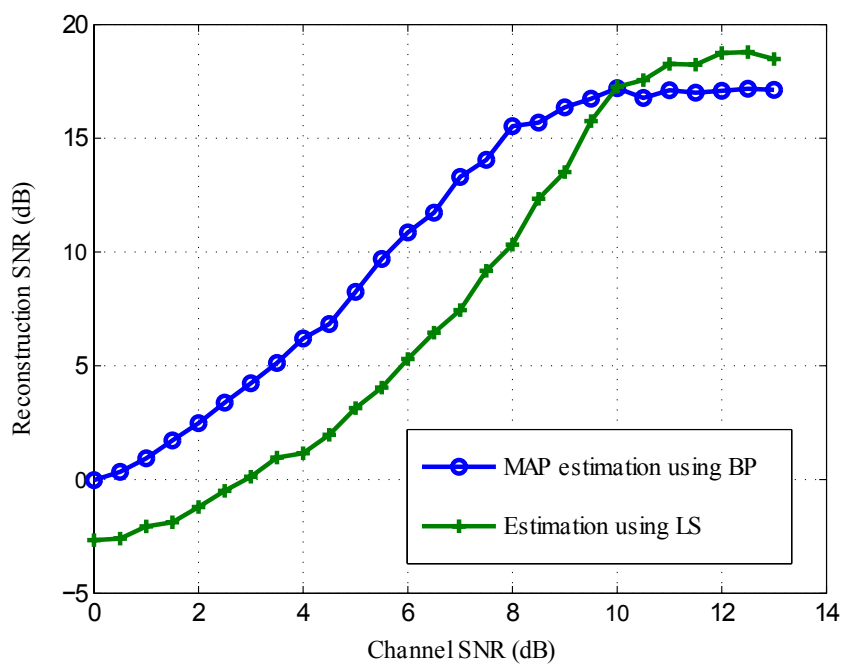

Fig. 6. The reconstruction SNR as a function of the channel SNR.

using BP reaches more than $5 d B$ in terms of the reconstruction SNR for a channel SNR equal to $6 d B$. For a channel SNR greater than $10 d B$ the LS estimator performs better, its gain is about $2 d B$ in reconstruction SNR.

\section{CONCLUSION}

In this work we have presented an implementation of the MAP estimation based on BP to estimate the input signal of an OFB from noisy subbands. The experimental results show that when the channel is noisy, this approach performs better in terms of reconstruction SNR than classical least-squares reconstruction.

The final version of this paper will evaluate the performance of GAMP in the considered context and consider OFBs with longer impulse responses, to evaluate the impact of the sparsity of the matrix representing the OFB.

\section{ACKNOWLEDGMENTS}

This work has been partly supported by the ANR CPP Project. M. Kieffer is partly supported by the Institut Universitaire de France.

\section{REFERENCES}

[1] C. E. Shannon, "A mathematical theory of communication," Bell Syst. Tech. J., vol. 27, pp. 379-423, 1948.

[2] A. K. Katsaggelos and F. Zhai, Joint Source-Channel Video Transmission, Morgan Claypool, 2007.

[3] H. Bölcskei and F. Hlawatsch, "Oversampled filterbanks: Optimal noise shaping, design freedom and noise analysis," in Proc. IEEE ICASSP., Munich, 1997, vol. 3, pp. 2453-2456.

[4] Z. Cvetković and M. Vetterli, "Oversampled filter banks," IEEE trans. Sig. Proc., vol. 46, no. 5, pp. 1245-1255, 1998.

[5] B. Champagne Q.-G. Liu and D. K. Ho, "Simple design of oversampled uniform DFT filter banks with applications to subband acoustic echo cancellation," Signal Processing, vol. 80, pp. 831-847, 2000.

[6] F. Labeau, J.C. Chiang, M. Kieffer, P. Duhamel, L. Vandendorpe, and B. Mack, "Oversampled filter banks as error correcting codes: theory and impulse correction," IEEE trans. Sig. Proc., vol. 53, no. 12, pp. 4619 - 4630, 2005.

[7] G. R. Redinbo, "Decoding real-number convolutionnal codes: Change detection, Kalman estimation," IEEE trans. Inf. Th., vol. 43, no. 6, pp. 1864-1876, 1997.

[8] G. R. Redinbo, "Wavelet codes: Detection and correction using Kalman estimation," IEEE trans. Sig. Proc., vol. 57, no. 4, pp. 1339-1350, 2009.

[9] V. K. Goyal, M. Vetterli, and N. T. Thao, "Quantized overcomplete expansions in $\mathbb{R}^{n}$ : Analysis, synthesis, and algorithms," IEEE Trans. Inf. Th., vol. 44, no. 1, pp. 16-31, 1998.

[10] M. Kieffer M. Abid and B. Pesquet-Popescu, "Consistent reconstruction of the input of an oversampled filter bank from noisy subbands," in Proc. EUSIPCO, 2011, Barcelona.

[11] S. Rangan, "Estimation with Random Linear Mixing, Belief Propagation and Compressed Sensing," arXiv:1001.2228v2 [cs.IT], 18 May 2010.

[12] S. Rangan, "Generalized approximate message passing for estiamation with random linear mixing," arXiv:1010.5141v1 [cs.IT], 25 Oct 2010.

[13] F. R. Kschischange and H.-A. Loeliger B. J. Frey, "Factor graphs and the sum-product algorithm," IEEE Trans. Inf. Th., vol. 47, no. 2, pp. 498-519, 2001.

[14] H.-A. Loeliger, "An Introduction to Factor Graph," IEEE Signal Processing Magazine, vol. 04, pp. 28-41, 2004.

[15] D.L. Donoho, A.Maleki, and A. Montanari, "Message passing algorithm for compressed sensing," arXiv:0907.3574v1 [cs.IT], 2009.

[16] Willian E. Ryan and Shu Lin, Channel Codes, Cambridge University Press, 2009.

[17] P. P. Vaidyanathan, Multirate Systems and Filterbanks, Prentice-Hall, Englewood-Cliffs, NJ, 1993. 